\title{
Modelización del proceso de degradación de envases metálicos para bebida, en el suelo, en el agua y en la interacción agua-suelo(*)
}

\author{
I. Rieiro*, V. Triviño*, T. Gutiérrez*, J. Muñoz* y M.T. Larrea**
}

\begin{abstract}
Resumen
En este estudio se evalúa la contaminación metálica que se produce a lo largo del tiempo al depositar en el suelo hojalata y hoja de aluminio, procedentes de envases comerciales de bebidas. Con el fin de evaluar el efecto del proceso de fabricación, se ha investigado el comportamiento del acero F111 y de la aleación de aluminio 3003, frecuentemente utilizados en la elaboración de estos envases. Para el estudio, se propone un diseño experimental que simula la degradación medioambiental a lo largo del tiempo de este tipo de envases cuando se depositan de forma incontrolada en el suelo. Los indicadores analíticos se han obtenido determinando la concentración de los metales mayoritarios en tres medios: agua, agua en presencia de suelo, y absorción-adsorción en suelo. Se ha desarrollado una metodología analítica para la determinación de la concentración de los elementos contaminantes mediante Espectrometría de Emisión Atómica con fuente de excitación ICP (ICP-OES). El método ha sido validado utilizando Materiales de Referencia (CRMs) de metales en suelo y agua; la precisión obtenida varía de 5,39 a 5,86\% y de 5,75 a 6,27\%, respectivamente, dependiendo del elemento analizado. El estudio estadístico llevado a cabo ha consistido en un análisis descriptivo seguido de un análisis factorial (modelo lineal general). La cuantificación metálica en los tres medios muestra que el suelo tiene un gran efecto inhibidor en la solubilidad de estos metales en agua. El proceso de fabricación de los envases para ambos materiales reduce la cesión metálica al medio.
\end{abstract}

Palabras clave Contaminación; Hojalata; Hoja de aluminio; Agua; Suelo; ICP-OES; Modelización.

\section{Degradation process modelization in of metallic drink containers, in soil, in water and in water-soil interaction}

\begin{abstract}
This study asses the environmental pollution by metal release that takes place during prolonged exposures when metallic drink containers are accidentally settle in the soil in a uncontrolled way, For comparative purposes, the F111 steel and the aluminium alloy 3003, widely used for the fabrication of these containers, are also considered. A experimental design is proposed to simulate the environmental pollution during prolonged exposures. Analytical indicators have been obtained determining the metallic concentration from three types of mediums; water, water in presence of soil, and absorption-adsorption in soil. An analytical methodology has been developed by Atomic Emission Spectrometry with ICP as exciting source (ICP-OES) for metallic quantification. The method was validated using Certified Reference Materials (CRMs) of soil and water and the precision obtained varies from 5.39 to $5.86 \%$ and from 5.75 to $6.27 \%$, respectively according to of the element studied. A statistical descriptive study followed by a factorial analysis (linear general model) has been carried out for the treatment of the experimental data packages. The metallic quantification for the three mediums shows that the soil inhibits metallic solubility in water. The process to make packages reduces in both cases their metallic cession.
\end{abstract}

Keywords

Pollution; Tin can; Aluminum can; Water; Soil; ICP-OES; Modelization.

\section{INTRODUCCIÓN}

La contaminación ambiental por metales, minerales o sus productos transformados es un hecho en gran medida atribuible al desarrollo tecnológico. Como consecuencia del incremento en la producción industrial se generan productos que una vez utilizados producen desechos. Estos desechos son depositados en muchas ocasiones de forma incontrolada sobre el suelo, lo que puede contribuir de forma grave a la

(•) Trabajo recibido el día 4 de abril de 2013 y aceptado en su forma final el día 5 de julio de 2013.

* Dpto. de Matemáticas. Facultad de Ciencias del Medio Ambiente. Universidad de Castilla-La Mancha, Avda. Carlos III s/n, 45071 Toledo.

** Dpto. de Metalurgia Física, Centro Nacional de Investigaciones Metalúrgicas (CSIC). Avda. Gregorio del Amo 8 . 28040 Madrid, E-mail: m.larrea@cenim.csic.es 
alteración del medio ambiente ${ }^{[1]}$.

El suelo es una de las matrices más complejas que existen debido a las variables físicas, químicas y biológicas que lo constituyen, Por otra parte, los metales en su interacción con el suelo pueden establecer uniones con las diferentes fracciones que lo componen: en unos casos serán de carácter lábil, y por tanto susceptibles de solubilizarse en el agua, en otros, de constituirse en biodisponibles dentro de la cadena trófica y en otros casos podrán permanecer en el suelo fuertemente fijado al mismo ${ }^{[2-4]}$.

Las prácticas más comunes que contribuyen a la degradación del suelo se deben a pesticidas, residuos domésticos y desechos industriales. Dentro de los residuos domésticos se encuentran los envases metálicos utilizados para bebidas, conocidos vulgarmente como latas.

La producción de los envases metálicos ha sufrido una evolución importante en su proceso de fabricación desde 1938. La relevancia de este tipo de envases va unida al cambio de costumbres sociales impulsadas por el desarrollo económico, lo que se traduce en una incorporación importante de estos materiales a los residuos antrópicos ${ }^{[5]}$.

Los materiales de estos envases más generalizados en su uso por sus características intrínsecas son los de hojalata y de hoja de aluminio, tanto por su capacidad para mantener en óptimas condiciones el contenido como por su capacidad de reciclabilidad, siendo este último aspecto de suma relevancia debido a su potencial contaminante. A pesar de que la Unión Europea sitúa el 50\% de reciclado como una buena meta, todavía queda un importante porcentaje de residuos de envases de bebida que se encuentran incontrolados.

La utilización de un material u otro en la elaboración de las latas varía según la disponibilidad de cada uno de estos materiales en los diferentes países. En España se viene utilizando de forma más generalizada la hojalata y en el norte de Europa la hoja de aluminio. En el caso de la hoja de aluminio los envases se reciclan mediante tecnologías que utilizan corrientes de Foucault y los de hojalata se separan del resto de residuos mediante separación magnética con imanes y posterior fusión en hornos para recuperar la materia prima.

El proceso de elaboración de estos envases metálicos está en continua evolución con objeto de conseguir mayores prestaciones; esto genera la necesidad de incorporar elementos adicionales a las materias primas de partida, los cuales hay que tener en cuenta desde la perspectiva no solo de su posible contaminación del medio, sino de su interacción con los elementos de degradación procedentes de las materias primas.
Cuando un envase metálico se deposita en el suelo sufre un proceso de degradación de todos sus componentes y éstos van a interaccionar en un primer momento con el suelo, con el agua presente en el suelo y con el agua de la capa freática. En esta interacción se producen procesos de absorción-desorción y de solubilidad. Estos procesos son complejos y hay que estudiarlos por separado y en su conjunto debido a sus interacciones, que afectan a la solubilidad y por tanto requieren, para una mejor comprensión de los procesos que se producen, la elaboración de un balance entre los tres sistemas presentes donde se pueda evaluar la solubilidad en agua, la absorción/adsorción en suelo y la solubilidad en agua en presencia de suelo.

El suelo, por si solo no actúa como un gran vector dispersor de la contaminación sino que más bien lo hace como sumidero para los contaminantes, dependiendo de su capacidad de retención o de amortiguación, pero en combinación con otros factores como el agua y el aire, sí que puede convertirse en vector.

Los metales mayoritarios, que forman parte de la matriz de los envases de bebida más utilizados, son el hierro y el aluminio, presentando este último una mayor reactividad ${ }^{[6]}$, lo que implica que su superficie sufra un proceso de pasivado, que puede afectar tanto su lixiviación en agua como su absorción en el suelo.

Todos los procesos de interacción entre el elemento contaminante y el medio se producen en la naturaleza a temperaturas que vienen marcadas por el clima y suceden en periodos de tiempo variable.

La solubilidad de los elementos constituyentes de los envases metálicos de bebida en el agua pueden verse afectada por la presencia de un vector complejo como es el caso del suelo. Así mismo la adsorción-desorción en el suelo, puede modificarse dinámicamente por la complejidad de los elementos absorbidos en su interacción con los elementos constituyentes del suelo. Es muy difícil a priori prever estos comportamientos ${ }^{[7]}$.

La estimación de forma individualizada de los procesos de cesión metálica de las materias primas y de las latas frente a los vectores suelo, agua y agua-suelo permite establecer las diferencias entre el comportamiento de las materias primas y de las mismas una vez que han sido sometidas al proceso de fabricación. Este conocimiento identificará si las diferentes fases llevadas a cabo en el proceso de fabricación tales como: inertización, soldadura, estañado y serigrafiado, tienen una influencia determinante en la degradación de estos materiales.

Los efectos de los procesos ambientales en la naturaleza sobre estos materiales tendrán que ser considerados en el diseño de experimentos que contemplará todos los factores a estudiar, simulándolos para condiciones extremas. 
De las diferentes técnicas de análisis para determinar el contenido de los elementos solubles en agua y absorbidos-adsorbidos en suelos cabe destacar la Espectroscopía de Emisión con fuente de excitación ICP (ICP-OES), por ser una técnica multielemental con buena precisión y exactitud y presentar límites de detección suficientemente bajos ${ }^{[8]}$.

La estadística descriptiva, como una herramienta necesaria en una primera instancia, permite obtener una aproximación básica del comportamiento de la distribución de los datos obtenidos por ICP-OES. Esta información derivada de la estadística descriptiva se confirma mediante contrastes paramétricos ( $t$-student y ANOVA), o en su caso análisis factoriales de varios factores. Posteriormente, con dichos datos, se pueden elaborar modelos matemáticos explicativos que permitan, al menos en el marco de balances de materia, controlar el proceso. Es interesante poder tener, al menos respecto de las variables de modelización de tiempo y temperatura, capacidades predictivas.

Si bien existen publicaciones ${ }^{[9}$ y 10$]$ sobre la contaminación que producen los elementos contaminantes considerados, no se ha encontrado en la bibliografía ningún estudio sobre los efectos de los envases.

El objetivo principal de este trabajo es evaluar la contaminación metálica que se produce a lo largo del tiempo al depositar en el suelo envases de bebida de hojalata y de hoja de aluminio. Para lograr este objetivo se realizará un diseño de experimentos para determinar los elementos que presentan: 1) solubilidad en agua, 2) adsorción/absorción en suelo y 3 ) solubilidad en agua en presencia de suelo. Todo ello a temperatura elevada y con agitación para obtener la máxima reactividad y a diferentes tiempos para evaluar la tendencia del proceso con el fin de simular las condiciones más extremas que se puedan producir en la naturaleza.

Como objetivo secundario de este estudio se determinará la influencia que el proceso de fabricación tiene en la degradación de estos envases, para lo cual se aplicará el mismo diseño experimental a las materias primas que los constituyen.

Para determinar estos elementos con precisión y exactitud, en los materiales propuestos se ha desarrollado una metodología analítica mediante espectrometría de emisión con fuente de ionización ICP.

\section{PROCEDIMIENTO EXPERIMENTAL}

\subsection{Materiales}

- Agua desionizada Milli-Q Plus de la firma Millipore (Bedford, MA, USA).
- Suelo de retamar arenoso (80-90\% de arena) neutro, $(\mathrm{pH} 6,4)$ con estructura débil.

- Material de Certificado de suelo: SO-2 perteneciente al horizonte $\mathrm{B}$ de un podsol férrico-húmico (Canada Centre for Mineral and Energy Technology).

- Material Certificado de agua: SRC 1643e (Nacional Institute of Standards \& Techology).

- Envase de hojalata.

- Envase de hoja de aluminio.

- Acero F 111.

- Aleación de aluminio 3003.

- Malla textil.

- $\mathrm{HNO}_{3} 65 \%, \mathrm{HClO}_{4} 60 \%$ y $\mathrm{HF} 40 \%$ (Merck "Pro-análisis").

- $\mathrm{Na}(\mathrm{OH})$ 0,01 M (Panreac).

- Bombas de digestión cerradas (Savillex).

\subsection{Instrumentación}

- Balanza (Marca: Mettler, modelo: AJ50).

- pHmetro (Marca Orión, modelo 720A + advanced ISE $/ \mathrm{pH} / \mathrm{mv} / \mathrm{ORP})$.

- Baño termostático con agitación (Marca: Clifton, modelo: NE5-28 Shaker Baths; 1050W; $50-60 \mathrm{~Hz} ; 220-240 \mathrm{~V})$.

- Estufa (0-200 ${ }^{\circ} \mathrm{C}$, Marca Venticell, modelo: Ecocell lím.).

- Espectrómetro de emisión con fuente de excitación ICP (Inductively Coupled Plasma Optical Emission Spectrometry) de la firma PerkinElmer, modelo: Optima 3200 DV (Palo Alto, CA, EE.UU.).

- Paquete estadístico SPSS 15,0.

\subsection{Procedimiento operativo}

En la figura 1 se recogen todas las etapas experimentales del estudio.

Se realizaron un total de 30 ensayos por triplicado con las cuatro muestras (hojalata, acero, hoja de aluminio y aleación de aluminio) en agua a $\mathrm{pH}$ neutro (similar al agua de lluvia) en presencia y ausencia de suelo, a una temperatura de $50^{\circ} \mathrm{C}$ con agitación, para acelerar las reacciones, y a tres tiempos distintos: 8,24 y $72 \mathrm{~h}$ respectivamente. En la tabla I se recogen los 10 ensayos para cada tiempo con el contenido y el indicador de medida de cada uno de ellos. Se incluyen los blancos correspondientes a cada ensayo. Se han considerado los siguientes blancos en cada caso:

- B1 para la medida de la solubilidad en agua.

- B2 para la medida de la solubilidad del agua en presencia de suelo. 


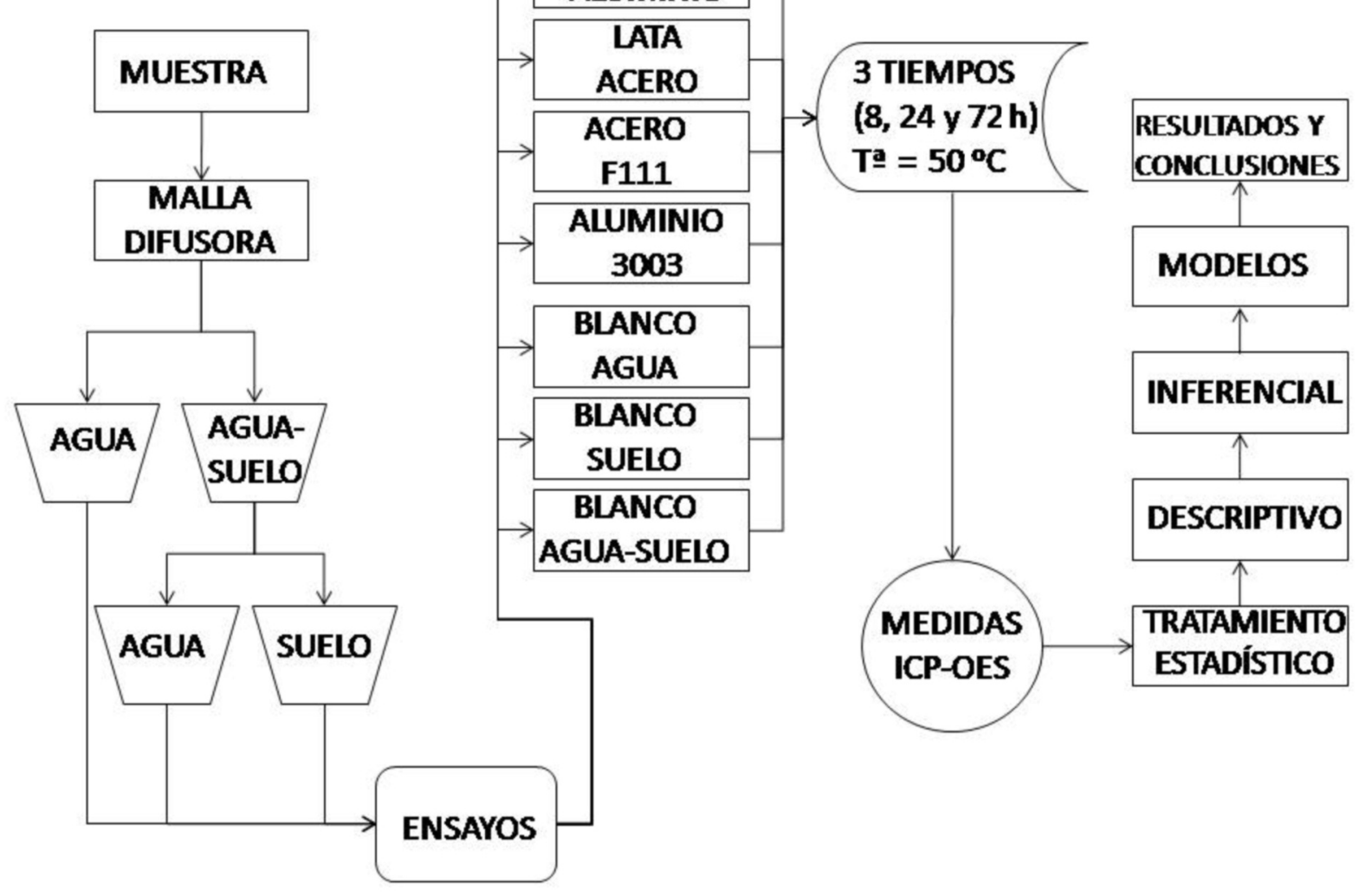

Figura 1. Diagrama de flujo con todas las etapas del proceso.

Figure 1. Flow chart with description off all process steps.

- B3 la medida de la absorción/adsorción del suelo después de filtrar el agua.

Para preparar cada ensayo se obtuvieron virutas $(<3 \mathrm{~mm})$ de las muestras. Se pesó en la balanza $7,0 \pm 0,5$ g de cada muestra y se introdujo, para cada ensayo, en un recipiente textil con una malla inferior al tamaño de las virutas.

El agua destilada de resistividad $>18 \mathrm{M} \Omega \mathrm{cm}$ se ajustó con el pHmetro a pH 7 con $\mathrm{Na}(\mathrm{OH})$ $0,01 \mathrm{M}$.

Se muestreó un suelo con $10 \mathrm{~cm}$ de profundidad, correspondiente a un horizonte A y se homogeneizó.

Se introdujo cada muestra en el baño termostático con agitación a $50^{\circ} \mathrm{C}$, según la descripción recogida en la tabla I para cada uno de los tres tiempos, realizándose un total de 30 ensayos, cada uno de ellos por triplicado. Una vez realizado cada uno de los experimentos se retiró el recipiente textil y se filtró para separar el agua del suelo. Las muestras de agua se reservaron hasta ser medidas y respecto al suelo, con objeto de obtener una solución que pueda ser medida por la técnica de ICP-OES se pro-

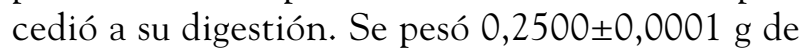

muestra de suelo y se introdujo en la bomba de digestión, se añadió $5 \mathrm{ml}$ de mezcla de ácidos (3:1, 5:0,5 de $\mathrm{HNO}_{3}, \mathrm{HClO}_{4}$ y $\mathrm{HF}$ ), se cerró y se metió en la estufa a $105 \pm 0,5^{\circ} \mathrm{C}$ durante $12 \mathrm{~h}$. Una vez digerida la muestra se trasvasó a un matraz de $25 \mathrm{ml}$ enrasándola con agua desionizada.

Se consideraron los elementos aluminio y hierro para su cuantificación por formar parte de los materiales objeto de estudio. Para cada uno de los elementos se seleccionaron las líneas espectrales más sensibles sobre las que se llevó a cabo un estudio de interferencias espectrales. Con este fin se prepararon soluciones con todos los elementos, salvo el elemento objeto de estudio, con las concentraciones habitualmente presentes en las muestras, seleccionando para ello las tres líneas de emisión más sensibles con el propósito de seleccionar la línea analítica para cada elemento.

Se ha utilizado el material de referencia de suelo SO-2 y el de agua SRM 1643e con objeto de verificar la exactitud y precisión de las medidas por ICP-OES tanto en agua como en suelo. Las condiciones instrumentales se recogen en la tabla II. 
MODELIZACIÓN DEL PROCESO DE DEGRADACIÓN DE ENVASES METÁLICOS PARA BEBIDA, EN EL SUELO, EN EL AGUA Y EN LA INTERACCIÓN AGUA-SUELO DEGRADATION PROCESS MODELIZATION IN OF METALLIC DRINK CONTAINERS, IN SOIL, IN WATER AND IN WATER-SOIL INTERACTION

Tabla I. Descripción e indicador de cada ensayo

Table I. Description and indicator for each test

\begin{tabular}{|c|c|c|}
\hline Ensayo & Descripción & Indicador \\
\hline 1 & $100 \mathrm{ml}$ de $\mathrm{H}_{2} \mathrm{O}$ (Blanco 1 agua) & Contenido de aluminio y hierro del agua \\
\hline 2 & $100 \mathrm{ml}$ de $\mathrm{H}_{2} \mathrm{O}+7 \mathrm{~g}$ de acero & Solubilidad de hierro en agua \\
\hline 3 & $100 \mathrm{ml}$ de $\mathrm{H}_{2} \mathrm{O}+7 \mathrm{~g}$ de aluminio & Solubilidad de aluminio en agua \\
\hline 4 & $\begin{array}{l}100 \mathrm{ml} \text { de } \mathrm{H}_{2} \mathrm{O}+7 \mathrm{~g} \text { de envase } \\
\text { metálico de hierro (hojalata) }\end{array}$ & $\begin{array}{l}\text { Solubilidad del envase metálico de hierro } \\
\text { (hojalata) en agua }\end{array}$ \\
\hline 5 & $\begin{array}{l}100 \mathrm{ml} \text { de } \mathrm{H}_{2} \mathrm{O}+7 \mathrm{~g} \text { de envase } \\
\text { metálico de aluminio }\end{array}$ & $\begin{array}{l}\text { Solubilidad del envase metálico de } \\
\text { aluminio en agua }\end{array}$ \\
\hline 6 & $\begin{array}{l}100 \mathrm{ml} \text { de } \mathrm{H}_{2} \mathrm{O}+7 \mathrm{~g} \text { acero }+ \\
100 \mathrm{~g} \text { de suelo }\end{array}$ & $\begin{array}{l}\text { Solubilidad de hierro en agua, dependiente } \\
\text { del suelo y adsorción de hierro por parte } \\
\text { de éste }\end{array}$ \\
\hline 7 & $\begin{array}{l}100 \mathrm{ml} \text { de } \mathrm{H}_{2} \mathrm{O}+7 \mathrm{~g} \text { de aluminio } \\
+100 \mathrm{~g} \text { de suelo }\end{array}$ & $\begin{array}{l}\text { Solubilidad de aluminio en agua, } \\
\text { dependiente del suelo y adsorción de } \\
\text { aluminio por parte de éste }\end{array}$ \\
\hline 8 & $\begin{array}{l}100 \mathrm{ml} \text { de } \mathrm{H}_{2} \mathrm{O}+7 \mathrm{~g} \text { de envase } \\
\text { metálico de hierro (hojalata) }+ \\
100 \mathrm{~g} \text { de suelo }\end{array}$ & $\begin{array}{l}\text { Solubilidad de hierro del envase metálico } \\
\text { en agua, dependiente del suelo y } \\
\text { adsorción de hierro del envase metálico } \\
\text { (hojalata) por parte de éste }\end{array}$ \\
\hline 9 & $\begin{array}{l}100 \mathrm{ml} \text { de } \mathrm{H}_{2} \mathrm{O}+7 \mathrm{~g} \text { de envase } \\
\text { metálico de aluminio }+100 \mathrm{~g} \\
\text { de suelo }\end{array}$ & $\begin{array}{l}\text { Solubilidad del aluminio del envase } \\
\text { metálico en agua dependiente del suelo y } \\
\text { adsorción de aluminio del envase metálico } \\
\text { por parte de éste }\end{array}$ \\
\hline 10 & $\begin{array}{l}100 \mathrm{ml} \mathrm{de} \mathrm{H}_{2} \mathrm{O}+100 \mathrm{~g} \text { de suelo } \\
\text { (Blanco } 2 \text { y 3) }\end{array}$ & $\begin{array}{l}\text { Blanco agua con suelo: solubilidad de los } \\
\text { componentes del suelo en el agua y blanco } \\
\text { suelo contenido de los elementos en suelo. }\end{array}$ \\
\hline
\end{tabular}

\subsection{Metodología estadística}

Se ha trabajado sobre dos tipos de índices, uno absoluto (IR) y otro relativo (IC). El IR se construye restando a cada medida individual la de su blanco correspondiente, y es un índice absoluto porque aporta un valor neto de la concentración del elemento medido en el medio. El IC se construye dividiendo cada una de las medidas entre la medida del blanco correspondiente y la información que aporta es de magnitud del incremento o decremento en el medio respecto del blanco:

$$
\begin{aligned}
& \mathrm{IR}=\mathrm{Cm}_{\mathrm{i}}-\mathrm{Cb}_{\mathrm{i}} \\
& \mathrm{IC}=\mathrm{Cm}_{\mathrm{i}} / \mathrm{Cb}_{\mathrm{i}}
\end{aligned}
$$

donde $\mathrm{Cm}_{\mathrm{i}}$ es la concentración para cada medida i y $\mathrm{Cb}_{\mathrm{i}}$, la concentración en el blanco para cada medida.
Siguiendo los análisis clásicos ${ }^{[11-14]}$ de la estadística descriptiva, se realizan medidas de centralización y dispersión para cada uno de los tratamientos y se han tratado y representado gráficamente las evoluciones temporales de la concentración (en IR e IC).

Se llevan a cabo modelos lineales generales factoriales, (trifactoriales, bifactoriales y unifactoriales) ${ }^{[15]}$ con objeto de determinar la significación de los factores de influencia que se consideran de importancia en este estudio y sobre todo para valorar la significación estadística de su efecto sobre la variable medida (concentración) valorada en forma de índices resta (IR) o cociente (IC).

Todos los modelos a aplicar se considerarán para determinar la varianza explicada a través de las variables propuestas en este diseño. Dado que la estadística descriptiva utilizada no aporta toda la información imprescindible para los objetivos de esta investigación, ha sido necesario disponer de modelos con capacidad predictiva o al menos interpretativa sobre los 
Tabla II. Condiciones instrumentales del Espectrómetro de Emisión Óptica ICP-OES

\author{
Table II. Operating conditions for the Optical Emission ICP \\ Spectrometer
}

\begin{tabular}{lc}
\hline Parámetro & Valor \\
\hline Modo visión del plasma & Axial y radial \\
Tiempo de medida & $2 \mathrm{~s}$ \\
Réplicas de las medidas & 6 \\
Radio frecuencia & $40 \mathrm{MHz}$ \\
RF Potencia incidente & $1.300 \mathrm{~W}$ \\
Caudal de Ar plasmógeno & $15 \mathrm{~L} \cdot \mathrm{min}^{-1}$ \\
Caudal de Ar de nebulización & $0,6 \mathrm{~L} \cdot \mathrm{min}^{-1}$ \\
Caudal de Ar auxiliar & $1 \mathrm{~L} \cdot \mathrm{min}^{-1}$ \\
Caudal de aporte de muestra & $1 \mathrm{~mL} \cdot \mathrm{min}^{-1}$ \\
Diámetro del inyector & $2,0 \mathrm{~mm}^{-1}$ \\
Nebulizador & de flujo cruzado Perkin-Elmer \\
Cámara de nebulización & de doble paso Ryton Scoot \\
\hline
\end{tabular}

fenómenos de contaminación ambiental a analizar.

El objeto del estudio es buscar la explicación de la varianza de los índices resta y cociente considerados como variables a modelizar y analizar sus varianzas, que puede ser explicadas por determinados factores (cualitativos u ordinales). Son especialmente interesantes los posibles efectos de los niveles del factor o factores, y las interacciones entre factores cuando hay más de un factor.

Los objetivos del análisis factorial aplicado en el estudio son:

- Analizar la influencia de diferentes niveles de un factor en la media de la respuesta.

- Validar los procesos de modelización y su capacidad.

Para validar el análisis de varianza se debe verificar el cumplimiento de:

a) Homogeneidad en las medias o valor cero de los residuos de las mismas.

b) Igualdad de varianzas entre muestras. (Las causas de variabilidad no atribuibles a las muestras deben permanecer constantes durante el experimento).

c) Normalidad de los residuos.

El estudio se lleva a cabo sobre los residuos de los modelos. Los análisis factoriales se han realizado con los factores Metal, Estado y Tiempo con los niveles siguientes para cada factor: (A) metal (aluminio, hierro), (B) estado (materia prima, metal del envase) y (C) tiempo $(8,24$ y $72 \mathrm{~h})$ y las interacciones entre estos factores.

$$
\gamma_{i j k}=\mu+\alpha_{i}+\beta_{j}+(\alpha \beta)_{i j}+\varepsilon_{i j k}
$$

donde $\mu$ es el valor medio de la variable respuesta concentración, $\alpha_{i}$ es el efecto diferencial del factor estado, $\beta_{\mathrm{j}}$ es el efecto diferencial del factor tiempo y $\alpha \beta_{\mathrm{ij}}$ es el efecto de la interacción de los dos factores siendo finalmente $\varepsilon_{\mathrm{ijk}}$ el ruido o perturbación que no se controla.

Cuando un modelo bifactorial no cumple las condiciones de control se ha pasado al estudio del modelo unifactorial, que es simplemente:

$$
\gamma_{i, j}=\mu+\alpha_{i}+\varepsilon_{i, j}
$$

donde no solo desaparece el segundo factor, sino también la interacción.

En el experimento se han realizado tres réplicas para cada tratamiento. Los contrastes de los efectos de los factores son:

$\mathrm{H}_{0}=\alpha_{1}=\alpha_{2}=\ldots \ldots=\alpha_{n}$, todos los niveles son iguales, o los $\mathrm{n}$ - niveles presentan el mismo efecto.

$H_{1}=\exists \alpha_{1} \neq \alpha_{2}$, existen al menos dos niveles diferentes dentro del conjunto de los $n$ - niveles del factor.

Algunas de las verificaciones de las hipótesis de los modelos no solo son prescriptivas sino que su incumplimiento invalida totalmente el uso del mismo, otras únicamente lo limitan. Cuando no se cumpla la hipótesis de normalidad se descarta de manera absoluta el modelo, y además en muchas ocasiones no es posible su corrección. El incumplimiento de la condición de homocedasticidad se puede evitar en ocasiones con una corrección mediante transformaciones logarítmicas o usando las más generales de Box-Cox. Es relevante el caso en el que el modelo, a pesar de no cumplir la homocedasticidad, presente 
valores altos del coeficiente de determinación $\mathrm{R}^{2}$, lo cual indica altos porcentajes de varianza de la variable respuesta explicados por el modelo ${ }^{[13}$ y 14] .

Así pues, con la finalidad de dejar establecido todo aquello que tiene una significación estadística, se han confeccionado modelos lineales generales para los procesos de la experimentación ${ }^{[15-17]}$.

La información estadística, analítica o gráfica obtenida de estos diseños, aplicados a los resultados de estos experimentos, proporciona una información sobre el comportamiento de los procesos y sobre su significación estadística. El hecho de aplicar los requisitos y condiciones de validez para estos modelos hace que sean muy restringidos los resultados científicamente evidenciados a través de los modelos estadísticos, aunque como consecuencia de ello los que resul$\tan$ significativos son concluyentes ${ }^{[15}$ y 18$]$.

Se han desarrollado experimentos ad-hoc para validar los aspectos más sorprendentes de las evidencias experimentales que aporta el análisis estadístico descriptivo ${ }^{[16-18]}$ En concreto, estos experimentos consistieron en las medidas de concentraciones de metales detectables presentes bien como microaleantes en las aleaciones utilizadas o bien como elementos provenientes de los procesados de los materiales de los envases o del propio suelo.

\section{RESULTADOS Y DISCUSIÓN}

El estudio de interferencias espectrales realizado ha permitido seleccionar como líneas analíticas, entre las tres más sensibles, aquéllas que se encontraron libres de interferencias (Tabla III). Se han calculado los límites de detección para cada una de ellas siguiendo los criterios de la guía EUROCHEM ${ }^{[19]}$.

Los resultados de los materiales de referencia utilizados para suelo y agua, (Tabla IV), muestran una buena concordancia con los valores certifica-
Tabla III. Líneas analíticas y límites de detección

Table III. Analytical lines and detection limits

\begin{tabular}{ccc}
\hline Elemento & $\begin{array}{c}\text { Línea analítica } \\
(\boldsymbol{\Lambda}=\mathbf{n m})\end{array}$ & $\begin{array}{c}\text { LD } \\
(\mathbf{m g} / \mathbf{L})\end{array}$ \\
\hline $\mathrm{Al}$ & 396,153 & 0,028 \\
$\mathrm{Fe}$ & 259,939 & 0,0062 \\
\hline
\end{tabular}

dos, estando sus coeficientes de variación entre $5,39-5,86 \%$ y entre $5,75-6,27 \%$ respectivamente para cada uno de los elementos, lo que indica que el análisis de los elementos, para los materiales considerados en los diferentes medios, presenta buena precisión y exactitud.

Con las concentraciones obtenidas para el hierro procedente del acero F111 y de la hojalata y para el aluminio procedente del aluminio 3003 y de la hoja de aluminio mediante la técnica de ICP-OES, en los diferentes medios, se han confeccionado los IR e IC de acuerdo al procedimiento descrito en el apartado 2.4. Dichos índices se recogen en las tablas V y VI respectivamente.

Los resultados con respecto a la solubilidad en agua del hierro procedente de los envases de hojalata y del acero F111 a los diferentes tiempos ensayados, se recogen en la figura 2 . En los valores obtenidos, expresados a través de los IR en $\mathrm{mg} / \mathrm{L}$, se observa que para la hojalata, a las $8 \mathrm{~h}$ no se ha empezado a solubilizar siendo ya apreciable a 24 h y de forma más marcada a las $72 \mathrm{~h}$; este fenómeno se corrobora a través de los IC que se presentan en la tabla $\mathrm{V}$.

Con respecto a la solubilidad en agua de este mismo elemento procedente del acero F111, a los dos primeros tiempos la solubilidad detectable es nula y solo aumenta ligeramente para el tiempo de expo-

Tabla IV. Índices IR e IC para el hierro procedente del acero F111 y de la hojalata para
diferentes tiempos

Table IV. IR and IC index for iron from steel F111 and from tin can at different times

\begin{tabular}{lcccccc}
\hline Elem. & $\begin{array}{c}\text { SO-2 Valor } \\
\mathbf{c e r t i f i c a d o} \\
(\boldsymbol{\mu g} / \mathbf{g})\end{array}$ & $\begin{array}{c}\text { SO-2 } \\
\text { Valor } \\
\text { encontrado } \\
(\boldsymbol{\mu g} / \mathbf{g})\end{array}$ & $\begin{array}{c}\text { Precisión } \\
\mathbf{C V} \\
(\%)\end{array}$ & $\begin{array}{c}\text { SRM 1643e } \\
\text { Valor } \\
\text { certificado } \\
(\boldsymbol{\mu g} / \mathbf{k g})\end{array}$ & $\begin{array}{c}\text { SRM 1643e } \\
\text { Valor } \\
\text { experimental } \\
(\boldsymbol{\mu g} / \mathbf{k g})\end{array}$ & $\begin{array}{c}\text { Precisión } \\
\mathbf{C V}\end{array}$ \\
$\mathbf{( \% )}$ \\
$\mathrm{Al}$ & $8,01 \pm 0,18$ & $8,48 \pm 0,4$ & 5,86 & $138,33 \pm 8,4$ & $147,01 \pm 8,6$ & 6,27 \\
$\mathrm{Fe}$ & $5,56 \pm 0,16$ & $5,86 \pm 0,001$ & 5,39 & $95,7 \pm 1,4$ & $101,2 \pm 1,8$ & 5,75 \\
\hline
\end{tabular}


Tabla V. Índices IR e IC para el hierro procedente del acero F111 y de la hojalata para diferentes tiempos

Table V. IR and IC index for iron from steel F111 and from tin can at different times

\begin{tabular}{lccccccc}
\hline & \multicolumn{4}{c}{ Acero } & \multicolumn{5}{c}{ Hojalata } \\
\hline Experimento & $\begin{array}{c}\text { Tiempo } \\
\text { (h) }\end{array}$ & 8 & 24 & 72 & 8 & 24 & 72 \\
& IR & 0,257 & 0 & 0,652 & 0 & 1,646 & 13,161 \\
Agua (mg/L) & IC & 41,428 & 1 & 105,098 & 1 & 265,556 & $2.122,741$ \\
& IR & 0,187 & $-22,269$ & 0,451 & $-1,614$ & $-23,509$ & $-0,946$ \\
Agua en presencia \\
de suelo (mg/L) & & & & & & & \\
Suelo (ìg/g) & IC & 1,066 & 0,209 & 1,27 & 0,427 & 0,165 & 0,433 \\
& IR & 0,188 & $-0,045$ & 0,189 & $-0,018$ & 0,236 & $-0,047$ \\
& IC & 1,113 & 0,977 & 1,095 & 0,989 & 1,121 & 0,977 \\
\hline
\end{tabular}

sición a 72 h. Estos comportamientos diferentes se podrían deber a que la materia prima sufre una oxidación superficial que inhibe su solubilidad en agua. Lo más destacable en este proceso es la información aportada por los IC para el caso del acero donde se observa una disminución de dicho índice a las $24 \mathrm{~h}$ con respecto a las $7 \mathrm{~h}$ para luego aumentar considerablemente a $72 \mathrm{~h}$. Este hecho podría ser debido a un proceso electroquímico debido a la presencia de los elementos aleantes y que no se manifiesta en la hojalata, por la falta de contacto con el agua al estar la materia prima recubierta por los elementos introducidos en los procesos de inertización y que actúan como barrera tanto frente al agua, retrasando la cinética de solubilización como decreciendo el fenómeno electroquímico observado en la materia prima. Estos efectos de los elementos aleantes de la materia prima con el tiempo van perdiendo levemente su actividad como se constata observando el incremento de la solubilidad y el del IC a las $72 \mathrm{~h}$.

El efecto del suelo sobre la solubilidad en agua para estos mismos materiales se recoge en la figura 3. Se puede observar, a través de los índices IR, que el suelo cuando está presente con el agua ejerce un efecto inhibidor total sobre la solubilidad en agua en los tres tiempos considerados y para los dos materiales. Cabe destacar el efecto que se produce a las $24 \mathrm{~h}$ y que es de la misma intensidad para los dos materiales: acero F111 y hojalata. Este efecto es de difícil interpretación; no obstante con respecto al

Tabla VI. Índices IR e IC para el aluminio procedente del aluminio 3003 y de la hoja de aluminio para diferentes tiempos

Table VI. IR and IC index for aluminum from aluminum 3003 and from aluminum can at different times

\begin{tabular}{|c|c|c|c|c|c|c|c|}
\hline \multirow[b]{2}{*}{ Experimento } & \multirow[b]{2}{*}{ Tiempo (h) } & \multicolumn{3}{|c|}{ Aluminio 3003} & \multicolumn{3}{|c|}{ Hoja de aluminio } \\
\hline & & 8 & 24 & 72 & 8 & 24 & 72 \\
\hline Agua (mg/L) & $\begin{array}{l}\text { IR } \\
\text { IC }\end{array}$ & $\begin{array}{c}0,141 \\
5,02\end{array}$ & $\begin{array}{l}0,216 \\
7,703\end{array}$ & $\begin{array}{l}0,792 \\
28,29\end{array}$ & $\begin{array}{c}1,494 \\
53,359\end{array}$ & $\begin{array}{c}0,476 \\
17,002\end{array}$ & $\begin{array}{c}0,23 \\
8,221\end{array}$ \\
\hline $\begin{array}{l}\text { Agua en presencia } \\
\text { de suelo (mg/L) }\end{array}$ & $\begin{array}{l}\text { IR } \\
\text { IC }\end{array}$ & $\begin{array}{l}2,88 \\
1,471\end{array}$ & $\begin{array}{c}-54,167 \\
0,067\end{array}$ & $\begin{array}{l}-0,799 \\
0,831\end{array}$ & $\begin{array}{l}-4,63 \\
0,243\end{array}$ & $\begin{array}{c}-54,111 \\
0,068\end{array}$ & $\begin{array}{c}-3 \\
0,365\end{array}$ \\
\hline Suelo (ìg/g) & $\begin{array}{l}\text { IR } \\
\text { IC }\end{array}$ & $\begin{array}{c}0,36 \\
1,065\end{array}$ & $\begin{array}{l}0,265 \\
1,043\end{array}$ & $\begin{array}{l}-0,165 \\
0,975\end{array}$ & $\begin{array}{l}2,103 \\
1,378\end{array}$ & $\begin{array}{l}0,144 \\
1,023\end{array}$ & $\begin{array}{l}-0,89 \\
0,864\end{array}$ \\
\hline
\end{tabular}


Fe en agua (IR)

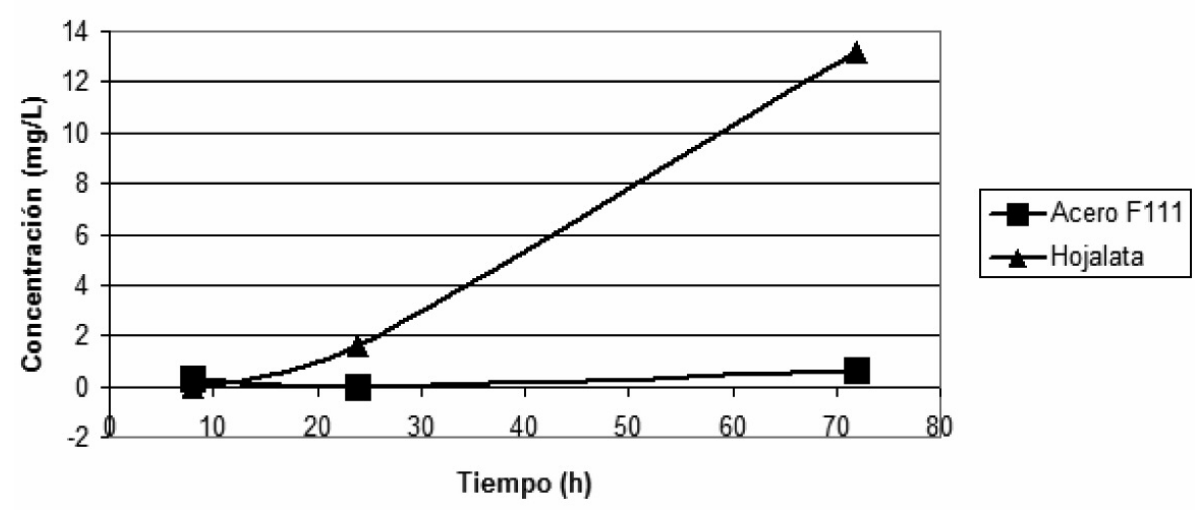

Figura 2. Solubilidad en agua del hierro procedente del acero F111 y de la hojalata.

Figure 2. Iron solubility in water from steel F111 and from tin can.

objetivo del trabajo, que es el de la cesión de los materiales estudiados, puede decirse también que para este tiempo no se produce cesión alguna al medio agua.

Para el caso de la absorción/adsorción de hierro en suelo, en la figura 4 se observa que el comportamiento es completamente inverso, entre el acero F111 y la hojalata. En el caso del acero, se detecta la misma cantidad a $8 \mathrm{~h}$ que a $72 \mathrm{~h}$, pero a las $24 \mathrm{~h}$ se produce el mismo efecto de bajada observado en estos materiales en agua sola. En el caso de la hojalata es justo al contrario, la concentración de hierro a 8 y $72 \mathrm{~h}$ es nula a los dos tiempos y menor que a $24 \mathrm{~h}$. Parece que en ambos casos se produce una variación a 24 h de la absorción/adsorción del suelo tanto por la presencia de hojalata como del acero aunque en sentido inverso. Hay que atribuir este comportamiento al efecto de las distintas etapas del procesado que presenta la hojalata, ya que es el único elemento diferenciador con respecto al acero. Podríamos por tanto interpretar que el suelo presenta una mayor absorción-adsorción del hierro procedente de la materia prima que del procedente del envase para 8 y $72 \mathrm{~h}$, lo cual es coherente cuando se constata que la solubilidad de la materia prima en agua en presencia de suelo es mayor a esos tiempos; sin embargo, a $24 \mathrm{~h}$ en el suelo se produce una desorción que con el tiempo vuelve a recuperar el suelo.

\section{Fe en agua en presencia de suelo (IR)}

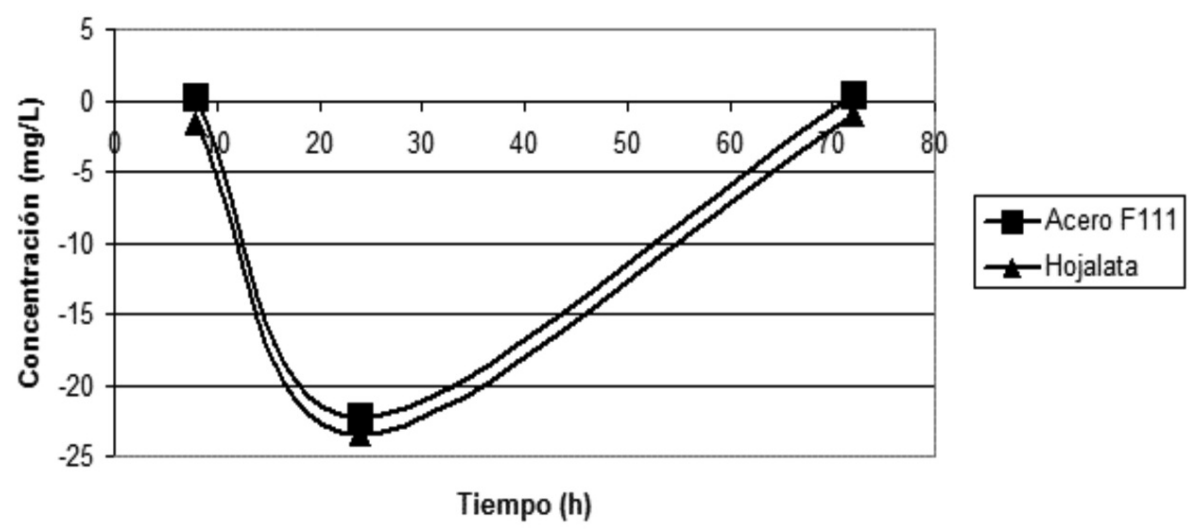

Figura 3. Solubilidad en agua en presencia de suelo del hierro procedente del acero F111 y de la hojalata.

Figure 3. Iron solubility in water with soil from steel F111 and from tin can. 


\section{Fe en suelo (IR)}

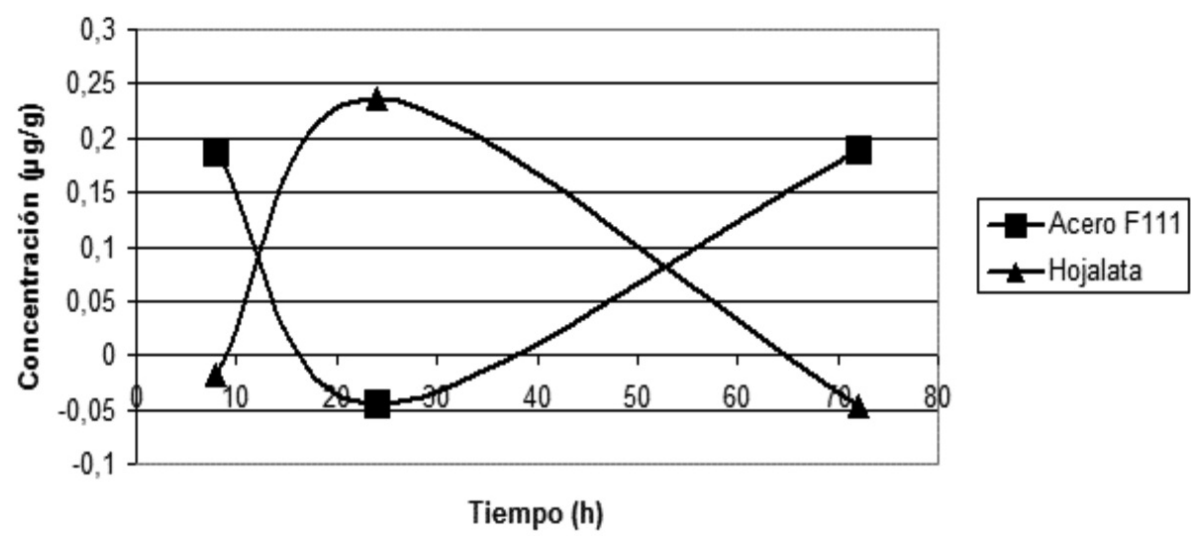

Figura 4. Absorción/adsorción en suelo del hierro procedente del acero F111 y de la hojalata.

Figure 4. Iron absortion/adsortion in soil from steel F111 and from tin can.

Al observar la tendencia de los IC se constata que para los tres tiempos son muy próximos a 1 (Tabla V). Esto quiere decir que en magnitud, las diferencias con respecto del blanco son nimias y no relevantes, aunque manifiesten una significación por los órdenes de magnitud. Debemos discernir en este caso la significación de la relevancia, lo que requiere un contraste de medias para poder decir que son diferentes.

Confirmado que efectivamente existen esas diferencias por ser estadísticamente significativas, en el caso de la hojalata se podría establecer un balance, porque lo que disminuye en el agua en presencia de suelo a $24 \mathrm{~h}$, aumenta en el suelo a ese mismo tiempo. Sin embargo, en el caso del acero, a $24 \mathrm{~h}$ se está produciendo esa disminución en los dos medios. En este sentido al ser el efecto en los dos casos similar y dado que el suelo es un medio altamente complejo, debido a las múltiples reacciones en su seno, no se puede establecer este balance en el marco de este estudio.

En el caso de los envases de aluminio, como se observa en la figura 5 , la solubilidad de aluminio en agua procedente del aluminio 3003 se comporta de forma esperada, esto es, aumenta con el tiempo de exposición. No es así en el caso de la hoja de aluminio. En este caso, la solubilidad del aluminio va dis-

\section{Al en agua (IR)}

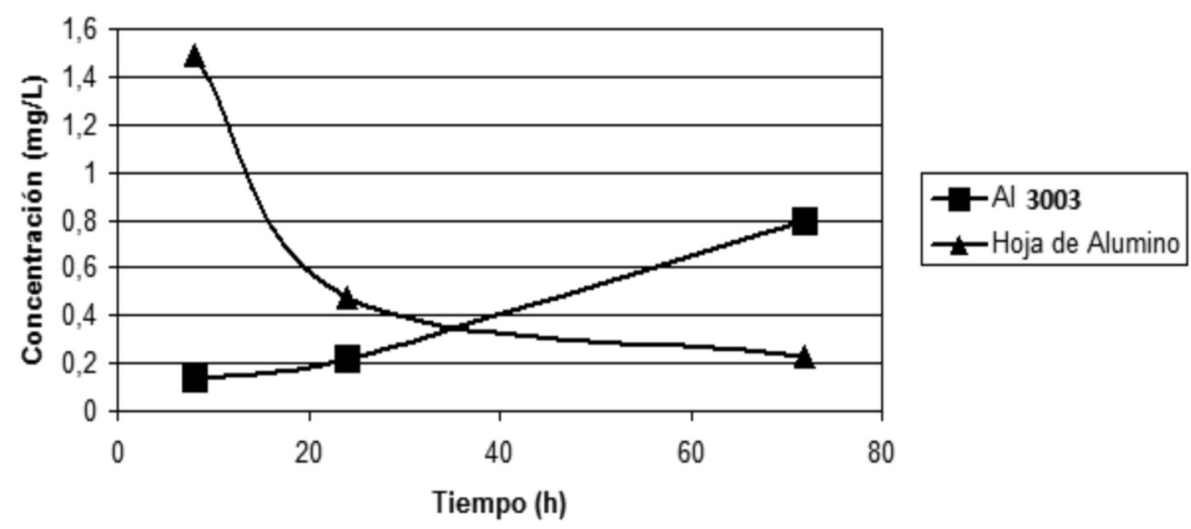

Figura 5. Solubilidad en agua del aluminio procedente del aluminio 3003 y de la hoja de aluminio.

Figure 5. Aluminum solubilility in water from aluminum 3003 and from aluminum can. 


\section{Al en agua en presencia de s uelo (IR)}

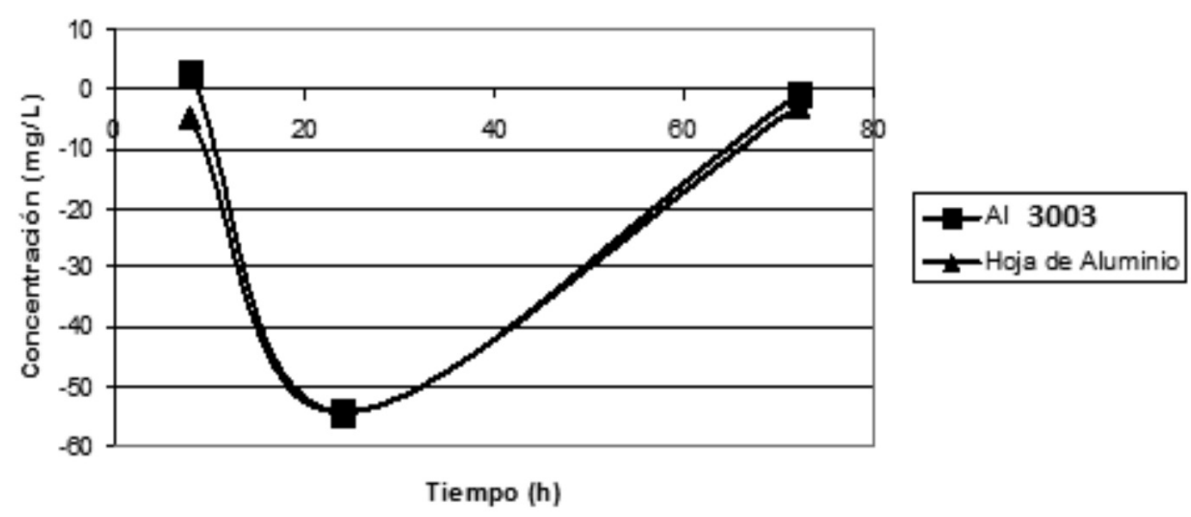

Figura 6. Solubilidad en agua en presencia de suelo del aluminio procedente del aluminio 3003 y de la hoja de aluminio.

\section{Figure 6. Aluminum solubility in water with soil from aluminum 3003 and from aluminum can.}

minuyendo conforme aumenta el tiempo que ha estado expuesto el material al agua.

Para 8 y 24 h, la solubilidad del aluminio es mayor en el caso de la hoja de aluminio. Los índices cociente son de magnitud considerable en todos los casos, luego se puede afirmar que lo que se detecta de aluminio no es casual, sino significativo.

El hecho de que la solubilidad del aluminio disminuya con el tiempo en el caso de la hoja de aluminio solo puede ser debido, por tanto, a los elementos presentes por el proceso de fabricación de las latas, que ejercen con el tiempo un efecto inhibidor de la solubilidad en agua, posiblemente por la formación de una capa de óxido de aluminio sobre la hoja de aluminio, pasivado, que con el tiempo va inhibiendo la solubilización inicial.

En el caso de la solubilidad del aluminio en agua en presencia de suelo (Fig. 6) se observa que la solubilidad del aluminio tiene prácticamente el mismo comportamiento que tenía la solubilidad del hierro del acero y de la hojalata. A 8 y $72 \mathrm{~h}$, para los dos materiales, la solubilidad es muy pequeña, y a $24 \mathrm{~h}$ de nuevo aparece la disminución drástica en la solubilidad, siendo negativa. Esto volvería a indicar que el suelo presenta un efecto inhibidor total sobre la solubilidad del aluminio en agua para los dos materiales, aluminio 3003 y hoja de aluminio y superior al ejercido sobre el acero y la hojalata, lo que puede ser debido a la mayor reactividad del aluminio.

Los valores del índice cociente, que representan el aumento o disminución respecto del blanco, son mucho más bajos que en el caso en el que no se añadió suelo al experimento, por lo que corrobora la afirmación, (al igual que ocurría para el hierro), de que la solubilidad del aluminio se ve disminuida drásticamente por la presencia de suelo.

En el caso de la absorción/adsorción del aluminio en suelo (Fig. 7) se observa que en ambos materiales, la evolución de la absorción/adsorción del aluminio con el tiempo es inversa: a mayor tiempo, es menor. Este descenso es más pronunciado en el caso de la hoja de aluminio. A $8 \mathrm{~h}$ la cantidad de aluminio en el suelo es mayor cuando se trata del material procesado, pero a 24 y $72 \mathrm{~h}$ es mayor su presencia en el caso de la materia prima. Solo a $72 \mathrm{~h}$ se detectan, en ambos casos, valores negativos en el índice resta, lo cual implicaría una pérdida de aluminio. Esto indicaría que el aluminio absorbido/adsorbido en el suelo con el tiempo vuelve al metal; esto se constata observando el incremento que se produce en el agua a este tiempo.

Los índices cociente vuelven a ser próximos a 1 (Tabla VI), y al igual que en el caso del hierro, esta tendencia es más acusada que en el caso del agua en presencia de suelo. Esto es debido a que el contenido de aluminio en el suelo es muy alto en comparación con el que puede ser aportado por los dos materiales experimentados.

Los resultados de la estadística descriptiva obtenidos son orientativos, no teniendo las consecuencias de un contraste estadístico.

La elaboración de modelos lineales generales de Diseño de Experimentos (ANOVA) unifactoriales, bifactoriales y trifactoriales, a partir de los datos experimentales permite afirmar que los modelos trifactoriales no son válidos por no presentar normalidad ni aleatoriedad de los residuos, por lo tanto se han desechado. 


\section{Al en suelo (IR)}

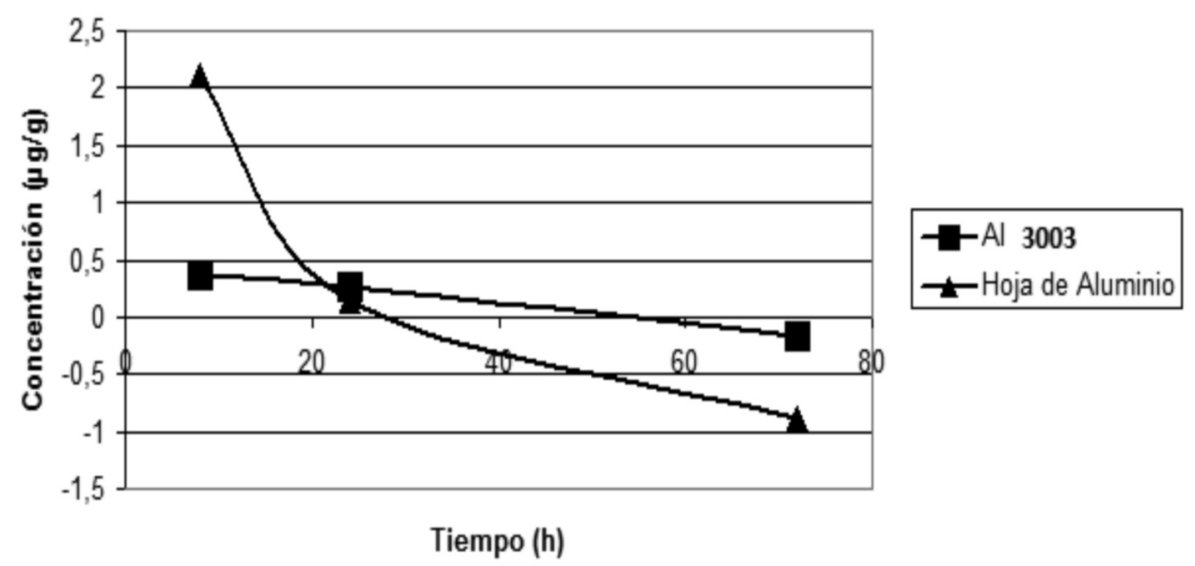

Figura 7. Absorción/adsorción en suelo de aluminio procedente del aluminio 3003 y de la hoja de aluminio.

Figure 7. Aluminum absortion/adsortion in soil from aluminum 3003 and from aluminum can.

Los modelos bifactoriales para el aluminio, no presentan homogeneidad de varianzas pero sí normalidad y homogeneidad de los residuos, además de valores del coeficiente de determinación de Pearson muy próximos a la unidad. Los modelos bifactoriales para el hierro no presentan homocedasticidad, normalidad ni homogeneidad por lo que hay que rechazarlos.

Debido a todo lo anterior los modelos bifactoriales no son de gran utilidad y la mayor parte de la información estadística válida la obtenemos de los modelos unifactoriales. Dado que estos modelos son muy numerosos, y que los resultados válidos se han extraído de una cantidad considerable de análisis unifactoriales, se ha sintetizado en la tabla VII.I, donde se puede observar que todos los resultados son válidos y significativos.

Se han estudiado los dos factores más importantes, el tiempo y el estado, en sus dos formas, materia prima o envase de bebida. Podemos observar la significación de la mayoría (todos excepto uno) de los contrastes que avalan las interpretaciones que nos aportaba la estadística descriptiva, lo cual quiere decir que cuando es significativo el factor en estudio es influyente.

En la tabla VII.I., se presentan dos zonas: (A) y (B). En la (A) se recogen los contrastes obtenidos sobre un único factor: el del proceso de fabricación, con sus dos niveles: metal sin procesar y metal procesado (envase). El contraste que se presenta en esta zona es sobre si hay o no hay diferencia significativa en las concentraciones del metal (sea éste hierro o aluminio), para cada tiempo $(8,24$ y $72 \mathrm{~h})$ y para cada medio (agua, agua en presencia de suelo y suelo). En cada celda cruce de todas estas situaciones se presenta el nivel de significación o p-valor. Al estar este valor por debajo de 0,05 se ha dado por válido el efecto diferencial de los niveles considerados sobre la concentración del metal en cada medio y cada tiempo.

En las celdas de la zona (B) se presenta el estudio de la significación de la influencia del factor tiempo; como este factor tiene tres niveles debe leerse de una forma diferente. Aquí los contrastes se hacen para un medio, agua, agua en presencia de suelo o suelo, al igual que en el bloque (A), pero luego se considera que sea sobre la materia prima o sobre el material manufacturado o envase para los tres niveles que entran en juego: 8,24 y $72 \mathrm{~h}$. Así un contraste significativo indica que hay al menos un tiempo diferente a los otros dos, pero podrían ser los tres tiempos diferentes entre sí, mientras que un contraste no significativo indica que las concentraciones en los tres tiempos son iguales. En estos casos en los que el número de niveles del factor en contraste es superior a dos, es obligado hacer un análisis "post-hoc" cuando el contraste es significativo, con la finalidad de ver cómo se agrupan los diferentes niveles en la diferencia de concentraciones de unos frente a otros.

Por lo observado en el segundo bloque (B) debemos pasar a la segunda parte de la tabla VII.I. En la tabla VII.II. se presentan los subgrupos "post-hoc" de los tiempos de exposición. La información recogida en el interior de las celdas indica, dado que el 
Tabla VII.I. Significaciones de los contrastes de igualdad de medias de los modelos unifactoriales ANOVA para los factores proceso de fabricación y tiempo

Table VII.I. Significances values for the statistical hypothesis test of the ANOVA one way for the manufacturing process and time factors

\begin{tabular}{|c|c|c|c|c|c|c|c|c|}
\hline \multirow{2}{*}{\multicolumn{2}{|c|}{$\begin{array}{l}\text { Subgrupos post- } \\
\text { hoc de tiempos } \\
\text { de exposición }\end{array}$}} & & \multicolumn{2}{|c|}{ Agua } & \multicolumn{2}{|c|}{$\begin{array}{c}\text { Agua en presencia } \\
\text { de Suelo }\end{array}$} & \multicolumn{2}{|c|}{ Suelo } \\
\hline & & & $\mathrm{Fe}$ & Al & $\mathrm{Fe}$ & $\mathrm{Al}$ & $\mathrm{Fe}$ & Al \\
\hline $\begin{array}{l}\text { Significación } \\
\text { de la influencia } \\
\text { del proceso } \\
\text { de fabricación } \\
\text { (A) }\end{array}$ & $\begin{array}{r}8 \mathrm{~h} \\
24 \mathrm{~h} \\
72 \mathrm{~h}\end{array}$ & $\begin{array}{l}\text { IR } \\
\text { IC } \\
\text { IR } \\
\text { IC } \\
\text { IR } \\
\text { IC }\end{array}$ & $\begin{array}{l}<0,05 \\
<0,05 \\
<0,05 \\
<0,05 \\
<0,05 \\
<0,05\end{array}$ & $\begin{array}{l}<0,05 \\
<0,05 \\
<0,05 \\
<0,05 \\
<0,05 \\
<0,05\end{array}$ & $\begin{array}{l}<0,05 \\
<0,05 \\
<0,05 \\
<0,05 \\
<0,05 \\
<0,05\end{array}$ & $\begin{array}{l}<0,05 \\
<0,05 \\
0,765 \\
<0,05 \\
<0,05 \\
<0,05\end{array}$ & $\begin{array}{l}<0,05 \\
<0,05 \\
<0,05 \\
<0,05 \\
<0,05 \\
<0,05\end{array}$ & $\begin{array}{l}<0,05 \\
<0,05 \\
<0,05 \\
<0,05 \\
<0,05 \\
<0,05\end{array}$ \\
\hline $\begin{array}{l}\text { Significación } \\
\text { de la influencia } \\
\text { del tiempo } \\
\text { (B) }\end{array}$ & $\begin{array}{c}\text { Materia } \\
\text { prima }\end{array}$ & $\begin{array}{l}\text { IR } \\
\text { IC }\end{array}$ & $\begin{array}{l}<0,05 \\
<0,05\end{array}$ & $\begin{array}{l}<0,05 \\
<0,05\end{array}$ & $\begin{array}{l}<0,05 \\
<0,05\end{array}$ & $\begin{array}{l}<0,05 \\
<0,05\end{array}$ & $\begin{array}{l}<0,05 \\
<0,05\end{array}$ & $\begin{array}{l}<0,05 \\
<0,05\end{array}$ \\
\hline
\end{tabular}

Tabla VII.II. Subgrupos para los contrastes que resultan significativos en el modelo unifactorial para el factor tiempo

Table VII.II. Subgroups for the significant contrasts in the in the one-way model for time factor

\begin{tabular}{|c|c|c|c|c|c|c|c|}
\hline \multirow{2}{*}{\multicolumn{2}{|c|}{$\begin{array}{l}\text { Subgrupos post- } \\
\text { hoc de tiempos } \\
\text { de exposición }\end{array}$}} & \multicolumn{2}{|c|}{ Agua } & \multicolumn{2}{|c|}{$\begin{array}{c}\text { Agua en presencia } \\
\text { de Suelo }\end{array}$} & \multicolumn{2}{|c|}{ Suelo } \\
\hline & & $\mathrm{Fe}$ & $\mathrm{Al}$ & $\mathrm{Fe}$ & Al & $\mathrm{Fe}$ & $\mathrm{Al}$ \\
\hline \multirow[t]{2}{*}{ Materia prima } & IR & $3^{(a)}$ & $3^{(\mathrm{a})}$ & $3^{(\mathrm{a})}$ & $3^{(a)}$ & $3\left({ }^{(a)}\right.$ & $2^{(b)}(8-24$ y 72$)$ \\
\hline & IC & $3^{(a)}$ & $3^{(\mathrm{a})}$ & $3^{(\mathrm{a})}$ & $3^{(a)}$ & $3^{(a)}$ & $2^{(b)}(8-24$ y 72$)$ \\
\hline \multirow[t]{2}{*}{ Envase de bebida } & $\mathrm{IR}$ & $2^{(b)}(8-24$ y 72$)$ & $3^{(a)}$ & $3^{(a)}$ & $3^{(a)}$ & $2^{(\mathrm{b})}(8-72$ y 24$)$ & $3^{(a)}$ \\
\hline & IC & $2^{\text {(b) }}(8-24$ y 72$)$ & $3^{(a)}$ & $2^{\text {(b) }}(8-72$ y 24$)$ & $3^{(a)}$ & $2^{\text {(b) }}(8-72$ y 24$)$ & $3^{(a)}$ \\
\hline
\end{tabular}

(a) Indica que hay tres subgrupos distintos, uno por cada tiempo: 8,24 y $72 \mathrm{~h}$

(b) Indica que hay dos subgrupos distintos, uno con dos tiempos y el segundo con el tiempo restante (entre paréntesis se indica la agrupación de los subgrupos).

contraste ha sido significativo, los subgrupos que hay dentro de los niveles posibles de 8,24 y $72 \mathrm{~h}$; éstos han sido 3 niveles, cuando a cada uno de los tres tiempos han dado concentraciones significativamente diferentes, y 2 niveles cuando dos de los tiempos se agrupan en un subgrupo y el tercero es diferentes (se indican las agrupaciones entre paréntesis).

Es obvio que en el caso de la parte (A) de la tabla VII.I no hacen falta subgrupos, pues al tratarse de dos niveles en caso de significación para cada uno de los niveles es un subgrupo. 


\section{CONCLUSIONES}

- La metodología desarrollada mediante ICP-OES permite la cuantificación de los metales tomados en consideración, con precisión y exactitud, tanto para los elementos solubles en agua como para los absorbidos/adsorbidos en suelo.

- La solubilidad del envase de hojalata en agua es creciente con el tiempo y muy superior a la observada en su materia prima. Con respecto a la hoja de aluminio y su matera prima, su solubilidad es muy inferior a la de la hojalata.

- El suelo, cuando está en presencia de agua, afecta a la cesión metálica de ambos envases y de sus materias primas ejerciendo un fuerte efecto inhibidor de su solubilidad. La absorción/adsorción que se produce en el suelo, siendo detectable para ambos envases y sus materias primas, resulta insignificante frente al contenido de estos metales en el suelo, confirmando así el papel amortiguador de este medio frente a la contaminación por estos metales.

- La estadística aplicada ha permitido garantizar la significación de la influencia de los factores tiempo y procesado sobre las concentraciones de hierro y aluminio, tanto en los dos envases como en sus materias primas.

- El análisis descriptivo de la estadística, junto con los modelos unifactoriales, ha permitido constatar, tanto a través de los índices resta como de los índices cociente, la evidencia significativa y objetiva de que, para todos los tiempos de exposición considerados, los dos envases de bebida metálicos estudiados (hojalata y hoja de aluminio), así como sus materias primas (acero F111 y aluminio 3003) tienen una influencia en las concentraciones medidas en todos los medios. Esto es, que los tiempos son un factor de influencia en la evolución de la solubilidad de los metales evaluada a través de las concentraciones respectivas, al mismo tiempo que se ha constatado que esta influencia no es lineal, lo cual implica para posteriores estudios una modelización y un diseño experimental mucho más complejo.

- El estudio de la influencia del factor de los tipos de estado (procesado, no procesado) en que se presentan los elementos aluminio y hierro permite asegurar de forma objetiva que el tipo de estado para ambos materiales y en todos los tiempos ensayados, presenta efectos estadísticamente significativos sobre la concentración en todos los medios.

\section{REFERENCIAS}

[1] C. Baird y M. Cann, Environmental Chemistry 4e, Ed. W.H. Freeman and
Company, New York, EE.UU., 2008, pp. 557 578 y $661-663$.

[2] D.C. Adriano, Trace elements TS in the Terrestrial Environment, Ed. Springer Verlag, New York, EE.UU., 1986, pp. 533-540.

[3] Má.I. Sánchez Bascones, Tesis Doctoral, Facultad de Ciencias, Universidad Valladolid, España, 2003, pp. 8-16.

[4] G. Sposito, The chemistry of soil, Oxford University Press, New York, EE.UU., 2008, pp. 195-218.

[5] w w w. lat a mania.coHYPERLINK http://www.latamania.com/.

[6] H.I. Scheinberg, Metals and their Compounds in the Environment: Occurrence, Analyses and Biological Relevance. Copper, Ed. Merian, E.VCH, New York, Basel, Cambridge, 1991, pp. 803-851.

[7] M. Juárez Sanz, J. Sánchez Andreu, y A. Sánchez Sánchez, Química del suelo y medio ambiente, Publisher Digitalia, 2006.

[8] P.W.J.M. Boumans, Inductively Coupled Plasma Atomic Emission Spectrometry, Part I: Methodology, Instrument and Performance, Part 1: Introduction to atomic emission spectrometry, pp. 358-417, Part 2: Applications: Metals and industrial Materials, 1987, pp. 4864, Ed. John Wiley \& Sons, 1987.

[9] R. Harichandan, S. Routroy, J.K. Mohanty y C.R. Panda, Enviromental monitoring Assessment 185 (2013) 3.503-3.516.

[10] Y. Crouau y E. Pinelli, Ecotoxicology and Environmental Safety 71 (2008) 643-649.

[11] N. James Miller y C. Jane Miller, Estadística y Quimiometría para Química Analítica, Ed. Prentice Hall-Pearson Educación, S.A., Madrid, España, 2002, pp. 1-41.

[12] R. Wayne Ott, Environmental Statistic and Data Analysis, Chapter 3: Analysis of observed data, pp. 46-71, Chapter 7: Normal Process, pp. 164-188, Chapter 8: Dilution of Pollutans, pp. 172-244, CRC Press, 1994.

[13] D.T. Kaplan, Statistical Modelling, CSIPP, 2009.

[14] C. Pérez, Estadística aplicada a través de Excel, Ed. Prentice Hall-Pearson Educación, S.A., Madrid, España, 2002, pp. 35-100 y 119-164.

[15] C. Pérez, Técnicas estadísticas con SPSS, Ed. Prentice Hall-Pearson Educación, S.A., Madrid, España, 2001.

[16] R. Serrano Gallego, Introducción al análisis de datos experimentales: Tratamiento de datos en Bioensayos, Capitulo 2: Estadística básica, pp. 19-42, Capítulo 4: El Análisis de la Varianza, pp. 67-82, Capitulo 7: Diseño Experimental y 
MODELIZACIÓN DEL PROCESO DE DEGRADACIÓN DE ENVASES METÁLICOS PARA BEBIDA, EN EL SUELO, EN EL AGUA Y EN LA INTERACCIÓN AGUA-SUELO DEGRADATION PROCESS MODELIZATION IN OF METALLIC DRINK CONTAINERS, IN SOIL, IN WATER AND IN WATER-SOIL INTERACTION

tratamiento de datos en Bioensayos, pp. 145160, Ed. Pub. de la Universitat Jaume, Castellón de la Plana, España, 2003.

[17] P.M. Berthouex y L.C. Brown. Statistics for Environmental Engineers, Ed. Lewis Publishers, Florida, EE.UU., 2002.

[18] D.C. Montgomery, Design and Analysis of Experiments, Chapter 3: Experiments with a
Single Factor: The Analisys of Variance, pp. 60-119, Chapter 5: Introduction to Factorial Designs, pp. 170-211, Model adequacy checking, pp. 76-86, Ed. Limusa - Wiley, Reino Unido, 2005.

[19] CITAC/Eurachem Working Group, Eurachem guide. Guide to Quality in Analytical Chemistry, 2002, pp. 23-29. 